\title{
Limits of precision in the Balmer lines spectroscopy lab
}

Timothy Roach ${ }^{1}$

${ }^{1}$ Physics Department, College of the Holy Cross, 1 College St., Worcester, MA 01610

Balmer lines spectroscopy can reach an impressive level of precision even with the common student spectrometer. Understanding the sources of error can help the instructor in guiding and assessing student work as well as in teaching about error analysis itself. We analyze the most significant contributions to error, both random and systematic, including approaches to minimize or measure them. This can be applied to teaching labs whether accuracy is $\sim 1 \%$ (introductory level) or better than $0.05 \%$ (in-depth advanced level).

\section{INTRODUCTION}

In this paper we examine the most significant sources of error that arise in measurement of the Balmer lines with a diffraction grating spectrometer, with a focus on applications to teaching a student laboratory course. An instructor's understanding of the causes and sizes of errors (both systematic and random) helps inform the experimental and analytical approaches for the lab, which can lead to better student understanding of their data.

We present our analysis of errors (or uncertainties, if you prefer) in the context of an example experiment, taken from a two-week investigation in our sophomore-level Modern Physics Laboratory course. We give what we hope is sufficient derivation so that our results can be easily adapted to other experimental approaches with similar equipment.

During the first week of the experiment, we review the basics of diffraction and the students learn how to measure the diffraction angles using a grating spectrometer [1]. They calibrate the instrument by measuring angles for light from a Hg discharge lamp, which has lines of known wavelength [2]. Their analysis gives a value for the grating constant $d$ and uncertainty $\Delta d$. In week two, they measure angles of the Balmer lines from a hydrogen lamp, from which they determine the wavelengths. These are compared qualitatively to the Bohr model and quantitatively to either the ionization energy or almost equivalently, to the Rydberg constant.

For a short intermediate or introductory lab, an accuracy of $1 \%$ may suffice and our analysis shows where the most significant errors might arise. However for a more in-depth lab like ours, errors can be brought down to $\sim 0.03 \%$ even using a student spectrometer and physical effects such as the reduced mass and index of refraction of air become significant and worthy of discussion and correction.

\section{DIFFRACTION THEORY}

A common type of spectrometer for the student lab uses a transmission diffraction grating, with light ideally arriving normal to the grating and beams due to constructive interference emerging as shown in Fig. 1. The light source is typically a gas discharge lamp from which some light is passed through a narrow slit aperture and then formed into a parallel beam by a collimation lens. In the normal-incidence situation, the diffraction angles are given as

$$
\sin \theta_{m}=\frac{m \lambda}{d}
$$

where $d$ is the diffraction grating constant (= inverse of the \# lines/meter).

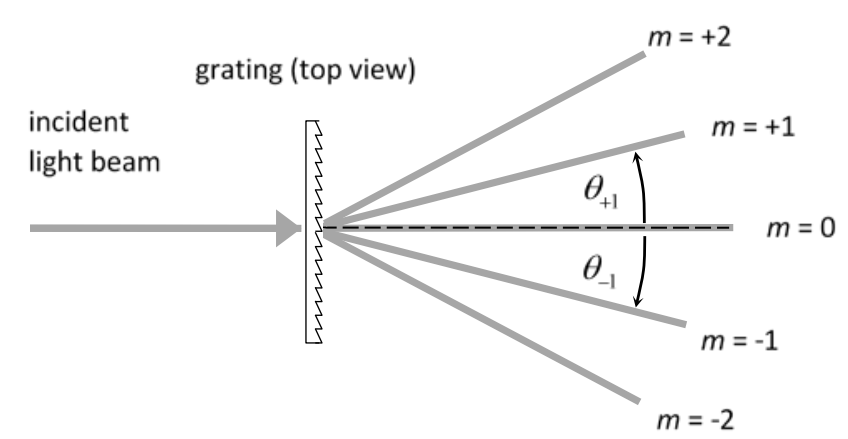

FIG. 1 Diffraction of monochromatic light into different orders (grating aligned for normal incidence).

A value for the wavelength can be determined from a measured diffraction angle as $\lambda=(d / m) \sin \theta_{m}$ from which we see that wavelength accuracy depends on the accuracy of $d$ and $\theta_{m}$.

If the grating is not arranged exactly normal to the incident light, then the diffraction condition is [3]

$$
\frac{m \lambda}{d}=\sin \theta_{m}+\sin \theta_{\mathrm{i}}
$$

where $\theta_{\mathrm{i}}$ is the angle of the incident beam relative to normal. Fig. 2 shows the situation for non-normal incidence, for outgoing beams $m=0, \pm 1$. Note that when $\theta_{\mathrm{i}}>0$ as shown, the $m=0$ beam is at a negative angle with respect to the normal, but equal in magnitude to $\theta_{\mathrm{i}}$.

Experimentally, it is difficult to determine the desired beam angle with respect to normal, $\theta_{m}$, in this situation. Many spectrometers give an angle reading $\varphi$ with respect to an angle origin that is never perfectly aligned with the grating or the incident light direction, but we can make use of the 
difference of two measurements, such as $\phi_{m}=\varphi_{m}-\varphi_{0}$. This is related to the desired angle and the misalignment as

$$
\phi_{m}=\theta_{m}+\theta_{\mathrm{i}} \text {. }
$$

This applies to both positive and negative values of $m$. (Note that in the drawing as shown, both $\phi_{-1}$ and $\theta_{-1}$ would have negative values).



FIG. 2 Angle definitions for case of a misaligned grating.

Usually one tries to arrange that $\theta_{\mathrm{i}}$ is so small that we can ignore its effect on calculation of the wavelength. In Section $B$ we look at the size of the error introduced and one approach for minimizing the error.

\section{MEASUREMENT ERRORS IN GRATING SPECTROSCOPY}

\section{A. Normal Incidence}

Let's first look at Eq. (1.1), applicable if the grating is well-aligned to be normal to the incident light. A first order expansion considering small uncertainties $\Delta \theta_{m}, \Delta d$ in the two variables gives a resulting change in calculated wavelength

$$
\Delta \lambda=\frac{\partial \lambda}{\partial d} \Delta d+\frac{\partial \lambda}{\partial \theta_{m}} \Delta \theta_{m}=\frac{1}{m} \sin \theta_{m} \Delta d+\frac{d}{m} \cos \theta_{m} \Delta \theta_{m}
$$

which we re-write as

$$
\frac{\Delta \lambda}{\lambda}=\frac{\Delta d}{d}+\cot \theta_{m} \Delta \theta_{m}
$$

The first term represents the relative uncertainty in the grating constant while the second term represents the relative uncertainty in the angle measurement. We will consider these two contributions separately.

\section{Error from angle measurement}

The uncertainty or error arising from measuring the diffraction angle we write as

$$
\left.\frac{\Delta \lambda}{\lambda}\right|_{\theta_{m}}=\cot \theta_{m} \Delta \theta_{m}
$$

or

$$
\left.\Delta \lambda\right|_{\theta_{m}}=\frac{d}{m} \cos \theta_{m} \Delta \theta_{m}
$$

Eq. (1.5) shows that the relative uncertainty in wavelength is proportional to $\cot \theta_{m}$, so the larger the angle the smaller the error in wavelength. However, students may not have much familiarity with cotangent and so lack instinct for the trend unless time is taken to examine it.

Eq. (1.6) shows that for a given wavelength, the absolute uncertainty in $\lambda$ decreases with increasing $m$. Thus there is a strong advantage to using higher orders, if they exist within the range of diffraction, restricted by $m \lambda / d<1$. The uncertainty decreases even more with angle as the cosine term decreases, but this is not so significant unless you go to angles larger than 45 degrees.

An example of the error propagation results is given in Table I, for light near the middle of the visible spectrum (specifically, wavelength $546 \mathrm{~nm}$, the green mercury line) and for a grating with 300 lines $/ \mathrm{mm}(d \simeq 3333 \mathrm{~nm})$. It assumes a measurement error $\Delta \theta_{m}=1$ minute of arc $=$ $0.29 \mathrm{mrad}$, the smallest measurement division on many student spectrometers. For a student with just a little practice, the typical angle measurement error is at least two times larger.

Table I: Wavelength error for different orders, caused by an error in measurement of diffraction angle of $1^{\prime}\left(\simeq 0.02^{\circ}\right.$ or $0.3 \mathrm{mrad}$ ).

\begin{tabular}{|c|c|c|c|}
\hline$m$ & $\begin{array}{c}\theta_{m} \\
(\mathrm{deg})\end{array}$ & $\begin{array}{c}\Delta \lambda \\
(\mathrm{nm})\end{array}$ & $\Delta \lambda / \lambda$ \\
\hline 1 & 9.4 & 0.96 & $0.175 \%$ \\
\hline 2 & 19.1 & 0.46 & $0.084 \%$ \\
\hline 3 & 29.4 & 0.28 & $0.052 \%$ \\
\hline 4 & 40.9 & 0.18 & $0.034 \%$ \\
\hline
\end{tabular}

\section{Error from grating constant (calibration error)}

The grating constant might be obtained from the manufacturer or as we do, by calibration using a known light source. The first term in Eq. (1.4) shows that uncertainty in the grating constant goes directly into uncertainty in wavelength,

$$
\left.\frac{\Delta \lambda}{\lambda}\right|_{d}=\frac{\Delta d}{d} .
$$

This is usually a systematic error, since one value of grating constant is used throughout an experiment. 


\section{B. Non-normal incidence: Grating Misalignment}

We suppose there is an unknown residual misalignment $\theta_{\mathrm{i}}$ and we make a measurement of the difference between the $m^{\text {th }}$ order and $0^{\text {th }}$ order beams, $\phi_{m}=\varphi_{m}-\varphi_{0}$. Using (1.3) the diffraction equation (1.2) becomes

$$
m \lambda / d=\sin \left(\phi_{m}-\theta_{\mathrm{i}}\right)+\sin \theta_{\mathrm{i}} .
$$

We might use $\phi_{m}$ to calculate wavelength assuming no misalignment, thence obtaining a value $\tilde{\lambda} \equiv(d / m) \sin \phi_{m}$. How large is the error caused by $\theta_{\mathrm{i}}$ ?

We begin by re-writing (1.7) to obtain

$$
\phi_{m}=\sin ^{-1}\left(\frac{m \lambda}{d}-\sin \theta_{\mathrm{i}}\right)+\theta_{i}=\sin ^{-1}\left(\sin \alpha-\sin \theta_{\mathrm{i}}\right)+\theta_{i}
$$

where for convenience of notation we introduce the quantity $\alpha$ defined by $\sin \alpha=m \lambda / d$. With these, our calculated wavelength becomes

$$
\tilde{\lambda}=(d / m) \sin \left[\sin ^{-1}\left(\sin \alpha-\sin \theta_{\mathrm{i}}\right)+\theta_{i}\right] .
$$

Eq. (1.8) can be expanded as a Taylor series in $\theta_{\mathrm{i}}$, a lengthy process which gives, to $2^{\text {nd }}$ order,

$$
\begin{aligned}
\tilde{\lambda} & =(d / m)\left[\sin \alpha+(\cos \alpha-1) \theta_{\mathrm{i}}+\frac{1}{2} \sin \alpha(2 \sec \alpha-1) \theta_{\mathrm{i}}^{2}\right] \\
& =\lambda\left[1+\csc \alpha(\cos \alpha-1) \theta_{\mathrm{i}}+\frac{1}{2}(2 \sec \alpha-1) \theta_{\mathrm{i}}^{2}\right]
\end{aligned}
$$

so that the relative error is

$$
\begin{aligned}
\Delta \lambda / \lambda & =(\tilde{\lambda}-\lambda) / \lambda \\
& =\csc \alpha(\cos \alpha-1) \theta_{\mathrm{i}}+\frac{1}{2}(2 \sec \alpha-1) \theta_{\mathrm{i}}^{2}
\end{aligned}
$$

This has a first-order error in the misalignment angle, with coefficient $\csc \alpha(\cos \alpha-1) \simeq \frac{1}{2}(m \lambda / d)$ for small $m \lambda / d$. Thus for a given misalignment the relative error increases roughly linearly with order and wavelength. An example of the wavelength error for several orders as a function of misalignment is given in Fig. 3.

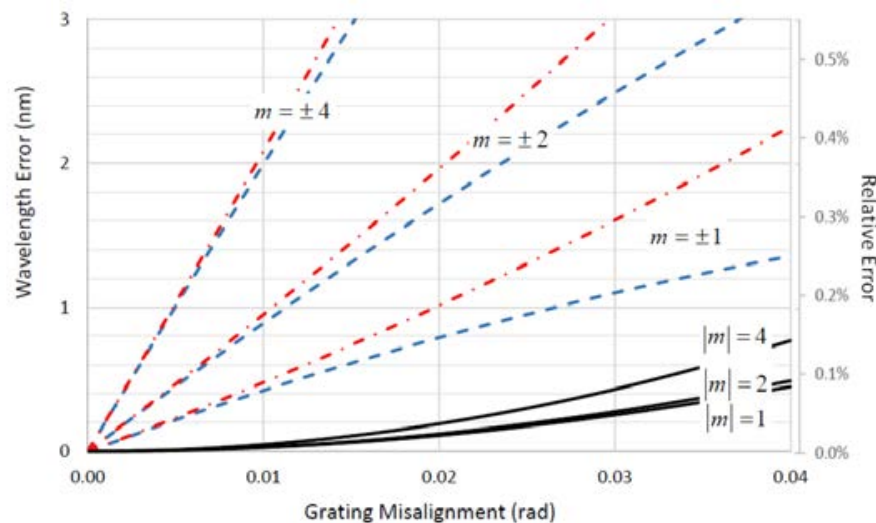

FIG. 3 Wavelength error from grating misalignment. Colored lines are measurement using single order (blue $m=+a$, red $m=-a$ ). Black lines are for combined orders $m=+a \&-a$, resulting in smaller errors. Values are for wavelength $546 \mathrm{~nm}, d=3333 \mathrm{~nm}$.
If there is a discernable (significant) grating misalignment then conceivably one could measure it essentially calibrate it - using a light source of known wavelengths and the model of Eq. (1.7). We use instead a simple and intuitive method to eliminate the first-order error, by combining two orders $m= \pm a$. We then compute

$$
\tilde{\theta}_{a} \equiv \frac{1}{2}\left|\varphi_{+a}-\varphi_{-a}\right|=\frac{1}{2}\left|\phi_{+a}-\phi_{-a}\right|
$$

and calculate a wavelength value $\tilde{\lambda}=(d / m) \sin \tilde{\theta}_{a}$. Combining this with Eq. (1.7) and once again expanding as a series to $2^{\text {nd }}$ order in $\theta_{\mathrm{i}}$ gives

$\tilde{\lambda}=(d / m)\left[\sin \alpha+\frac{1}{2} \sin \alpha \sec ^{2} \alpha \theta_{\mathrm{i}}^{2}\right]=\lambda\left[1+\frac{1}{2} \sec ^{2} \alpha \theta_{\mathrm{i}}^{2}\right]$ and

$$
\Delta \lambda / \lambda=(\tilde{\lambda}-\lambda) / \lambda=\frac{1}{2} \sec ^{2} \alpha \theta_{\mathrm{i}}^{2},
$$

which is only $2^{\text {nd }}$ order in misalignment. Interestingly but perhaps not surprisingly, the quadratic coefficient $\left(\sec ^{2} \alpha\right)$ is numerically very nearly equal to the quadratic coefficient in (1.9), the one-sided expansion: $(2 \sec \alpha-1)$. Both are $\sim 1-2$ for $\sin \alpha=m \lambda / d<0.7$ and increase only slowly in this range. A comparison of the errors arising from this computation method is shown in Fig. 3, where it is clear that it gives much smaller error due to misalignment than a onesided measurement. The error for $m=4$ becomes greater than the best possible precision error (Table $1,0.18 \mathrm{~nm}$ or $0.03 \%$ ) only for misalignments greater than about $0.02 \mathrm{rad}$.

\section{OTHER PHYSICAL EFFECTS}

The measurements described give wavelengths in air. If students compare their results to other measurements (for example, Balmer wavelengths from NIST), they should be sure to check if the values are for air or vacuum. If quantities relating to the structure of hydrogen are to be compared (for example, calculating the Rydberg constant from measured Balmer wavelengths) then the wavelengths should be converted to vacuum values first. These are related by $\lambda_{\text {vac }}=\lambda_{\text {air }} n_{\text {air }}$, where $n_{\text {air }} \simeq 1.00029$ is the index of refraction of air. Neglecting this would introduce a systematic relative error of $2.9 \times 10^{-4}$.

Another physical effect of similar size is that of the hydrogen nuclear mass, accounted for with the theoretical model of reduced mass. The Rydberg constant most often quoted is $R_{\infty}=1.0974 \times 10^{7} \mathrm{~m}^{-1}$, obtained essentially by precision measurement of the hydrogen Rydberg constant $R_{h}=1.0968 \times 10^{7} \mathrm{~m}^{-1}$ and "correcting" it, by approximately a relative amount $\simeq m_{e} / m_{p}=5.5 \times 10^{-4}$. 


\section{EXPERIMENTAL APPROACH NOTES}

\section{A. Angle measurement}

Students measure angles $\varphi_{ \pm m}$ and a typical random error for each measurement is about 2 minutes of arc. Using (1.10) they obtain the diffraction angle $\theta_{m}$, with a random error reduced by a factor of $1 / \sqrt{2}$, essentially due to "averaging" of the two angles. This means relative errors from actual student measurements in wavelength are about $40 \%$ larger than shown in Table 1.

The first measurement students do is of the $546 \mathrm{~nm}$ line of mercury as a practice, using the manufacturer's estimate of $d \simeq 1$ / $300 \mathrm{~mm}$ and they also carry out the error propagation analysis of (1.1) leading them to (1.6) and a tabulation as in Table 1. They discover that the difference between their calculated wavelength and known is much larger than can be explained by random error. This provides motivation for calibrating the grating themselves to find an accurate value for $d$. Furthermore, the observation that higher orders provide more accuracy leads us to choose to skip measuring the first order lines, since the accuracy is significantly lower. For $2^{\text {nd }}$ order to $4^{\text {th }}$ order, the typical student wavelength relative error from this method is better than $10^{-3}$ (about $0.05 \%$ to $0.1 \%$ ).

Larger errors regularly occur but usually fall into the "blunder" category. This happens with our apparatus when students mis-read the degree portion of the Vernier scale. The most common type of mistake puts one of the readings of $\varphi_{m}$ off by $0.5^{\circ}$, or an error of $0.25^{\circ}$ in $\theta_{m}$, several times the size of the typical random error.

\section{B. Grating \& Calibration}

We use a grating of 300 lines/mm because it allows students to measure several orders of visible light, giving a data set large enough to reduce statistical error, even if some lines are too faint to measure or if there are clear outliers that must be discarded. Alignment is done (usually by the instructor) by putting a mirror in place of the grating and adjusting it for retro-reflection, to within $\sim 10$ mrad.
For the calibration, our students measure four of the $\mathrm{Hg}$ lines (at approximately 436, 546, 577, and 579nm) in orders 2, 3, and 4, for a total of $N=12$ values. They fit to $a \lambda=d \sin \theta_{a}$ by least-squares with $d$ as an adjustable parameter [4]. Alternatively, one might compute values of $d$ and average them. In this case, one expects an accuracy improvement of $1 / \sqrt{N-1}$ from averaging, or a relative error of about $3 \times 10^{-4}$, assuming the same relative accuracy in $d$ as we derived for $\lambda$. In our lab, student error estimates (using a simplified chi-square type analysis for their leastsquares fit) are roughly this same size ( $\sim 0.5 \mathrm{~nm}$ to $3 \mathrm{~nm}$ out of 3300nm).

\section{Balmer lines measurement \& Rydberg constant}

Students measure the three longest wavelength Balmer lines, in orders 2,3 , and 4 (and $5^{\text {th }}$ order if possible). With three values for each wavelength, relative uncertainties of about $3 \times 10^{-4}$ or better ( 0.1 to $\left.0.2 \mathrm{~nm}\right)$ are often achieved.

The individual wavelengths are used to calculate values for the Rydberg constant and an average value ( $N \simeq 9$ ), with a relative random error $\sim 1.5 \times 10^{-4}$. We have them include the systematic error from the calibration, typically about twice this size, for a total relative error $\sim 5 \times 10^{-4}$. Careful students have uncertainties even smaller, making it worthwhile to include the corrections for reduced mass and index of refraction of air.

\section{CONCLUSION}

We have described in detail how measurement errors propagate and affect the results obtained from a common student grating spectrometer, applied to measurement of the Balmer lines. Systematic error from misalignment is reduced significantly by combining measurements on both sides of $m=0$. Random error can be reduced statistically by measuring several orders. Total error may be low enough that results are sensitive to small effects such as the reduced mass.

[1] For example, we use a PASCO Model SP-9268A and other similar older spectrometers but there may be others which are equally suitable.

[2] Sansonetti, C. J., Salit, M. L. \& Reader, J. Wavelengths of spectral lines in mercury pencil lamps. Appl. Opt. 35, 74-77 (1996).

[3] Optics, $4^{\text {th }}$ Edition (2002), p478, by E. Hecht. Because this is a transmission grating, we use a definition of incident angle with sign opposite to that given by Hecht, which is for a reflection grating.

[4] A similar but more elegant fitting model is described in The Covariance Matrix and Jacobian in Error Propagation, poster presentation by Bob Deserio, 2018 Proceedings of the Conference on Laboratory Instruction Beyond the First Year of College (BFYIII). 